\title{
Ettore Majorana's legacy to contemporary physics
}

\author{
R. Pucci ${ }^{*}{ }^{a b}$ F. S. Cataliotti, ${ }^{a c d e f}$ G. G. N. Angilella ${ }^{a b g}$ \\ ${ }^{a}$ Dipartimento di Fisica e Astronomia, Università di Catania, \\ Via S. Sofia, 64, I-95123 Catania, Italy. \\ ${ }^{b}$ CNISM, UdR Catania, I-95123 Catania, Italy. \\ ${ }^{c}$ LENS, Dipartimento di Fisica, Università di Firenze, \\ Via Nello Carrara, 1, I-50019 Sesto Fiorentino (FI), Italy. \\ ${ }^{d}$ Laboratorio CNR-INFM-MATIS, I-95123 Catania, Italy. \\ ${ }^{e}$ Istituto Superiore per la Formazione di Eccellenza, Università di Catania, \\ Via S. Nullo, 5/i, I-95125 Catania, Italy. \\ ${ }^{f}$ INFN, Laboratori Nazionali del Sud, I-95123 Catania, Italy. \\ INFN, Sez. Catania, I-95123 Catania, Italy. \\ E-mail: renato.pucciect.infn.it \\ E-mail: francesco.cataliottiect.infn.it \\ E-mail: giuseppe.angilellaect.infn.it
}

\begin{abstract}
To celebrate the centennial of Ettore Majorana's birth (1906-1938?), we survey the legacy of his scarce but fundamental scientific production to nearly all branches of physics, from atomic and molecular, to nuclear and subnuclear physics. In particular, we focus on a not so well known short communication by Majorana, where he critically discusses Fermi's solution of the celebrated Thomas-Fermi equation for electron screening in atoms and positive ions. We argue that some of Majorana's seminal contributions in molecular physics already prelude to the idea of exchange interactions (or Heisenberg-Majorana forces) in his later works on theoretical nuclear physics. We also describe a recent experiment on atomic confinement in magnetic traps, based on the ideas developed in Majorana's paper on the behaviour of atoms in a varying magnetic field. In all his papers, Majorana emphasized the symmetries at the basis of a physical problem, as well as the limitations, rather than the advantages, of the approximations of the method employed.
\end{abstract}

International Conference - Ettore Majorana's legacy and the Physics of the XXI century October 5-6, 2006, University of Catania, Italy

\footnotetext{
*Speaker.
} 


\section{Introduction}

Despite his scarce scientific production, Ettore Majorana's contributions to modern physics are vast and profound. His papers influenced the development of nuclear and subnuclear, as well as atomic and molecular physics, as we will emphasize in this contribution.

Certainly, Majorana's most famous and seminal papers are those entitled Teoria relativistica di particelle con momento intrinseco arbitrario (Relativistic theory of particles with an arbitrary intrinsic angular momentum) [1], Sulla teoria del nucleo (On the theory of the nucleus) [2], Teoria simmetrica dell'elettrone e del positrone (Symmetric theory of the electron and the positron) [3]. In particular, the latter work focussed on the so-called 'Majorana's neutrino'. Majorana's contributions to subnuclear and particle physics have been properly emphasized [4], and still nowadays provide the motivation to much fundamental research [5, 6].

In this note, we would like to reconsider three more papers by Majorana [7, 8, 9], both on atomic and molecular physics. We will show that his work on the molecular ion $\mathrm{He}_{2}^{+}[8]$ paved the way to his theoretical work on the exchange nuclear forces, the so-called Heisenberg-Majorana forces [2]. We will also try and emphasize his critical sense and great ability to catch the relevant physical aspects of a given problem, beyond his celebrated mathematical skills, as witnessed by contemporaries and colleagues who met him personally [10, 11, 12] (see especially Ref. [13] for more references).

On the other hand, Majorana's theoretical works have implicitly suggested experiments of fundamental atomic physics which, as noted by Majorana himself, could not be realized by means of the limited technology of his times. In particular, we will describe an experiment suggested by Majorana's work on the behaviour of an atom in the presence of a magnetic field [9], which has been recently carried out at the Quantum Information Laboratory of the Scuola Superiore of Catania, by employing modern techniques of atomic confinement in magnetic traps.

The paper is organized as follows. We review in some detail Majorana's approach to ThomasFermi equations in Section 2. In Section 3 we then describe an experiment recently carried out on atomic confinement in magnetic traps, based on Majorana's theoretical description of an atom in a varying magnetic field. Majorana's theory of the helium molecular ion (Section 4) is then presented as the basis for the exchange or Heisenberg-Majorana forces, later developed in nuclear theory (Section 6). In Section 5 we briefly review the discovery of the neutron. We summarize and give some concluding remarks in Section $\mathbf{7}$.

\section{Thomas-Fermi model}

The development of contemporary physics is dominated by the quest for symmetry. It was an apparent discrepancy between the transformation properties of Newton equations of dynamics and Maxwell equations of electrodynamics under a change of coordinates which led Einstein to lay the foundations of the theory of relativity, and to the unification of space and time [14]. On the other hand, it was the same Einstein [15], with de Broglie [16] among the others, who recognized another marked asymmetry - the asymmetry between particles and waves, - which would have been reconciled within quantum mechanics. 
Besides space-time and wave-particle symmetry, an important role is played by symmetries in matter. The simplest and most symmetric description of electrons in a metal is the so-called free electron, or jellium, approximation [17]. Such a model was introduced in the independent works of Thomas [18] and of Fermi [19], who treated electrons in an atom as a gas of independent particles with locally homogeneous density.

Within Thomas-Fermi approximation, the electronic cloud surrounding an atom is described in terms of a completely degenerate Fermi gas. Following Ref. [20], one arrives at a local relation between the electron density $\rho(\mathbf{r})$ at position $\mathbf{r}$ with respect to the nucleus, and the momentum $p_{\mathrm{F}}(\mathbf{r})$ of the fastest electron (Fermi momentum), as

$$
\rho(\mathbf{r})=2 \cdot \frac{4 \pi}{3} p_{\mathrm{F}}^{3}(\mathbf{r})
$$

where the factor of two takes into account for Pauli exclusion. In Eq. (2.1), the Fermi momentum $p_{\mathrm{F}}(\mathbf{r})$ depends on position $\mathbf{r}$ through the self-consistent potential $V(\mathbf{r})$ as

$$
p_{\mathrm{F}}^{2}(\mathbf{r})=2 m\left[E_{\mathrm{F}}-V(\mathbf{r})\right]
$$

where $E_{\mathrm{F}}$ is the Fermi energy, and $m$ is the electron mass. Fermi energy $E_{\mathrm{F}}$ is then determined via the normalization condition

$$
\int \rho(\mathbf{r}) d^{3} \mathbf{r}=N
$$

where $N$ is the total electron number, which equals the atomic number $Z$ for a neutral atom. Inserting Eq. (2.2) into Eq. (2.1), making use of Poisson equation, and introducing Thomas-Fermi screening factor $\phi$ through

$$
V(\mathbf{r})-E_{\mathrm{F}}=-\frac{Z e^{2}}{r} \phi(\mathbf{r})
$$

one derives the adimensional Thomas-Fermi equation for a spherically symmetric electron distribution,

$$
\frac{d^{2} \phi}{d x^{2}}=\frac{\phi^{3 / 2}}{x^{1 / 2}}
$$

where

$$
r=b x
$$

and $b$ sets the length scale as

$$
b=\frac{1}{4}\left(\frac{9 \pi^{2}}{2 Z}\right)^{1 / 3} a_{0}=\frac{0.8853}{Z^{1 / 3}} a_{0}
$$

with $a_{0}$ the Bohr radius.

Eq. (2.5) is 'universal', in the sense that the sole dependence on the atomic number $Z$ comes through Eq. (2.7) for $b$. Once Eq. (2.5) is solved, the self-consistent potential for the particular atom under consideration is simply obtained by scaling all distances with $b$. 


\subsection{Asymptotic behaviour of the solution to Thomas-Fermi equation}

Fermi tried to solve Eq. (2.5) analytically without success. On the occasion of his first meeting with Majorana, Enrico succintly exposed his model to Ettore, and Majorana got a glimpse of the numerical results he had obtained over a week time, with the help of a primitive calculator. The day after Majorana reappeared and handled a short note to Fermi, where he had jotted down his results. Majorana was amazed that Fermi's results coincided with his own.

How could Majorana solve Eq. (2.5) numerically in such a short time without the help of any calculator? Various hypotheses have been proposed. Did he find an analytical solution? At any rate, there are no physically acceptable analytical solutions to Eq. (2.5) in the whole range $0 \leq x<+\infty$. The only analytical solution,

$$
\phi(x)=\frac{144}{x^{3}}
$$

would have been found later by Sommerfeld in 1932 [21], and is physically meaningful only asymptotically, for $x \gg 1$.

The most likely hypothesis is probably that of Esposito [22], who, together with other authors [23], has found an extremely original solution to Eq. (2.5) in Majorana's own notes (see also Ref. [24]). The method devised by Majorana leads to a semi-analytical series expansion, obeying both boundary conditions for a neutral atom

$$
\begin{aligned}
\phi(0) & =1, \\
\phi(\infty) & =0 .
\end{aligned}
$$

In a recent work, Guerra and Robotti [25] have rediscovered a not well known short communication by Majorana, entitled Ricerca di un'espressione generale delle correzioni di Rydberg, valevole per atomi neutri o ionizzati positivamente (Quest for a general expression of Rydberg corrections, valid for either neutral or positively ionized atoms) [7]. In that work, perhaps in the attempt of improving the asymptotic behaviour of the solution to Thomas-Fermi equation, Ettore requires that the potential vanishes for a certain finite value of $x$, say $x_{0}$, both for neutral atoms and for positive ions. He writes the self-consistent potential as

$$
V(r)=\frac{Z e}{r} \phi+C,
$$

where, for an atom positively ionized $n$ times $(n=Z-N)$, the constant $C$ equals

$$
C=\frac{n+1}{b x_{0}} e,
$$

where

$$
b=0.47 \frac{1}{Z^{1 / 3}}\left(\frac{Z-n}{Z-n-1}\right)^{2 / 3} \AA,
$$

and the boundary conditions to Eq. (2.5) now read

$$
\begin{aligned}
\phi(0) & =1, \\
\phi\left(x_{0}\right) & =0, \\
-x_{0} \phi^{\prime}\left(x_{0}\right) & =\frac{n+1}{Z} .
\end{aligned}
$$




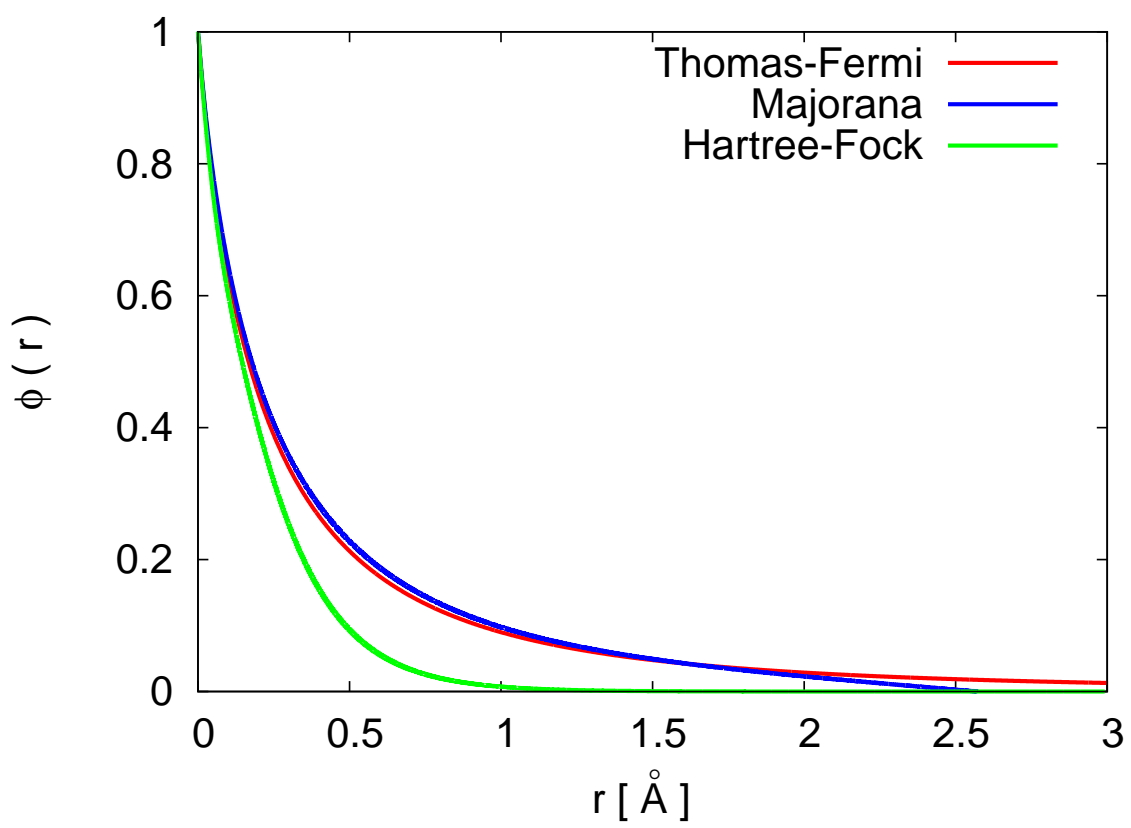

Figure 1: (Color online.) Thomas-Fermi screening factor $\phi$ for the self-consistent potential of a neutral Ne atom $(Z=N=10)$. Red line is Fermi's solution, blue line is Majorana's solution, while green line has been obtained within Hartree-Fock approximation.

One immediately notices that, due to the new boundary conditions, Eq. (2.10) does not reduce to Eq. (2.4) even for $n=0$, i.e. for a neutral atom. In other words, Majorana does not consider the potential $V(\mathbf{r})$ in a generic location of the electron cloud, but the effective potential acting on a single electron, thus excluding the interaction of an electron with itself.

Probably, owing to his profound critical sense (let us remind that his colleagues in the Panisperna group nicknamed him the 'Great Inquisitor'), Majorana must have not excessively relied on his own solution [22], which however reproduced the numerical solution of Thomas-Fermi equation quite accurately. Probably, Majorana was looking for a solution which should not decrease so slowly as $x \rightarrow \infty$, as Eq. (2.8) does.

In Fig. 1 we report Thomas-Fermi screening factor $\phi$ as a function of $r$ for a neutral Ne atom $(Z=N=10)$. The red line refers to Fermi's numerical solution, with boundary conditions given by $\mathrm{Eq}(\mathrm{s})$. (2.9), the blue line refers to Majorana's solution, with boundary conditions given by $\mathrm{Eq}(\mathrm{s})$. (2.13) with $n=0$, while the green line has been obtained within the Hartree-Fock approximation (see Ref. [26] for a derivation), and corresponds to the radial electron density shown in Fig. 2 as a red line (neutral Ne). As it can be seen, Majorana's solution introduces only a minor correction to Fermi's solution at finite $x$ values, but is strictly zero for $x \geq x_{0}$.

In his work on positive ions [27], Fermi considers a potential vanishing at a finite value $x=x_{0}$. However, instead of Eq(s). (2.13), he employs the boundary conditions

$$
\begin{aligned}
\phi(0) & =1, \\
-x_{0} \phi^{\prime}\left(x_{0}\right) & =\frac{n}{Z},
\end{aligned}
$$

which in particular imply Eq. (2.9b) in the case $n=0$, corresponding to a neutral atom. 


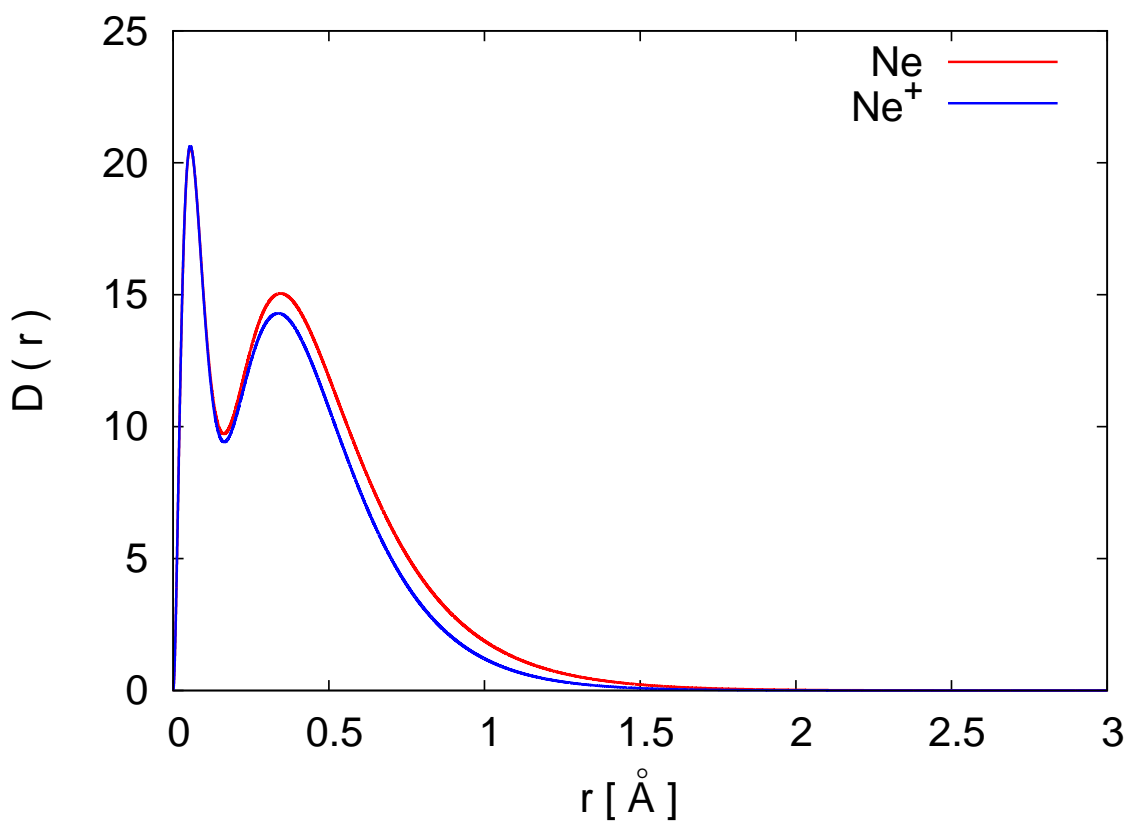

Figure 2: (Color online.) Radial electron density $D(r)=4 \pi r^{2} \rho(r)$ within Hartree-Fock self-consistent approximation for neutral $\mathrm{Ne}$ (red line) and singly ionized $\mathrm{Ne}^{+}$(blue line).

In Fig. 3, we again report Thomas-Fermi screening factor $\phi$ as a function of $r$ according to Fermi, Majorana, and Hartree-Fock, respectively, but now for a positively ionized $\mathrm{Ne}$ atom, $\mathrm{Ne}^{+}$ ( $Z=10, N=9, n=1)$. The Hartree-Fock solution corresponds to the radial electron density shown as a blue line in Fig. 2] (see Ref. [26] for a derivation). Majorana's solution again differs but marginally from Fermi's solution, but while for a neutral Ne atom Fermi's solution decreases too slowly, it decreases too rapidly for $\mathrm{Ne}^{+}$.

Here, we are not disputing whether Majorana's note, Ref. [7], should be considered as a 'full' paper [28], nor do we want to undervalue the importance of the contribution analyzed in Ref. [22]. We would rather like to emphasize that Majorana was conscious that his correction ${ }^{1}$ did not lead to substantial modifications to Fermi's solution of Eq. (2.5), including in the asymptotic limit $(x \gg 1)$ [31].

Ettore never published anything else on this subject.

\section{Majorana and atomic confinement in magnetic traps}

One of the most important contributions by Ettore Majorana to atomic physics is Ref. [9], which deals with the behaviour of oriented atoms in a varying magnetic field.

In the early 1930s, after the pioneering experiments by Stern and Gerlach [32] and by Phipps [33], researchers started investigating the behaviour of a magnetic dipole in a varying magnetic field. Güttinger [34], among the others, had studied the case of a motionless atom in the presence of a magnetic field varying with time. At variance with those studies, Majorana recognizes as more

\footnotetext{
${ }^{1}$ Flügge [29] erroneously attributes this correction to Amaldi. Probably, he was only aware of Fermi and Amaldi's final work, Ref. [30].
} 


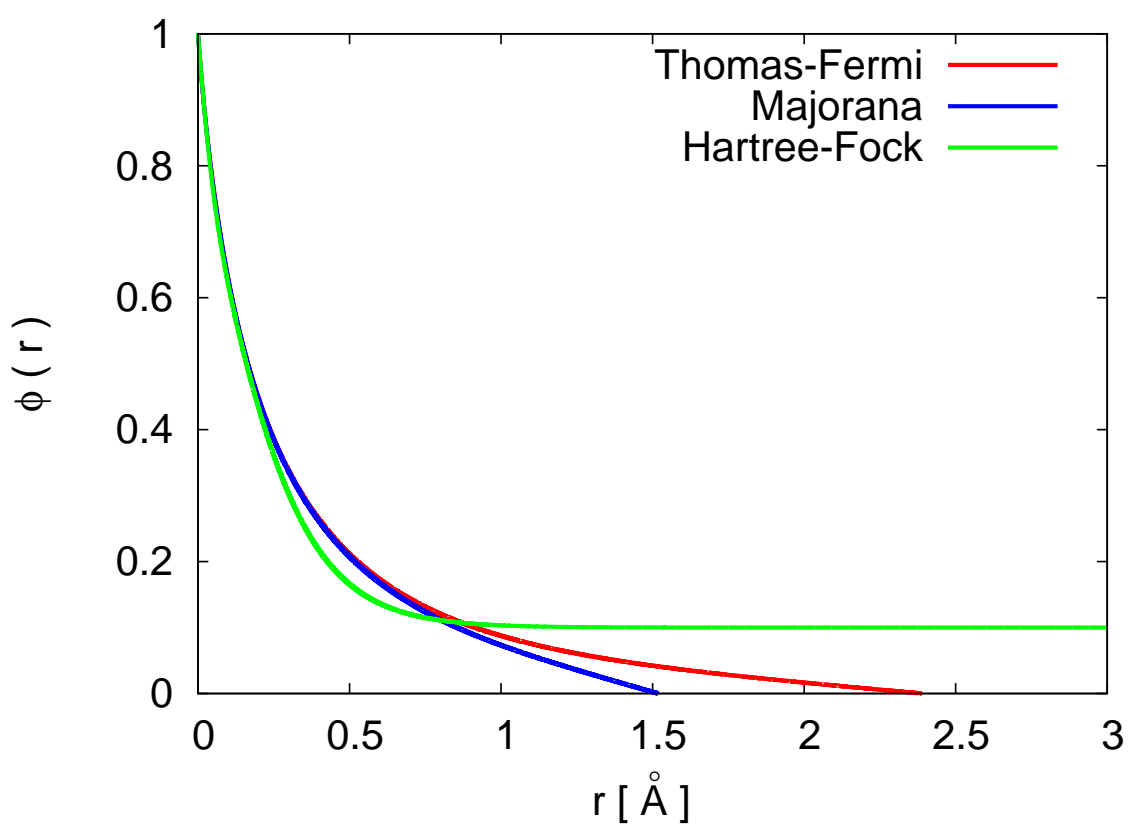

Figure 3: (Color online.) Thomas-Fermi screening factor $\phi$ for the self-consistent potential of the $\mathrm{Ne}^{+}$ion $(Z=10, N=9)$. Red line is Fermi's solution, blue line is Majorana's solution, while green line has been obtained within Hartree-Fock approximation.

physically relevant the case of an atomic beam propagating in a non-uniform, i.e. spatially varying, magnetic field. It is interesting to note that Majorana immediately realized that such an experiment would not be feasible in a laboratory at his times, since thermal beams would be characterized by relatively high velocity $\left(\sim 10^{5} \mathrm{~m} / \mathrm{s}\right)$ and relatively low alignment $(<1 \mathrm{~mm})$. An actual interest in this kind of experiments has been recently rekindled at the beginning of the 1990s, when laser cooling techniques and magnetic trapping of atoms became available, thus allowing to manipulate atoms with a velocity of some $\mathrm{cm} / \mathrm{s}$, with a spatial resolution of a few microns. The solution to the problem of the 'Majorana hole' paved the way to the experimental realization of Bose-Einstein condensation, which deserved the Nobel prize to its authors [35, 36].

In his 1932 work, however, Majorana [9] not only sets out the problem, but also provides it with a theoretical solution, employing for the first time the concept of 'Bloch sphere' and 'generalized Bloch sphere'. Actually, Felix Bloch himself, in his 1945 work with Isidor Rabi [37], makes extensive reference to Majorana's paper, emphasizing its fundamental importance for the solution of the problem. Remarkably enough, the title of Bloch and Rabi's paper [37] is almost literally the English translation of the title of Majorana's paper [9].

Besides solving the problem of an atom in a varying magnetic field, both classically and quantistically, Majorana proposes an innovative theoretical framework, which stimulated the development of a new and fruitful language for the description of a physical phenomenon. Such a theoretical framework is nowadays applied to nearly all field in physics, and affords an intuitive understanding of completely different phenomenologies, as advanced as quantum computation.

Let us focus briefly on the experimental implications of Majorana's work, especially as far as atomic cooling and magnetic trapping is concerned. To this aim, one makes use of the fact that the 
overall magnetic dipole of an atom adiabatically orients itself along an applied magnetic field, so as to minimize the potential energy in correspondence with the magnetic field's minima. The two simplest configurations to obtain a local minimum in the magnetic field are the quadrupole configuration, characterized by a linearly varying magnetic field along the three spatial directions, passing through zero at the origin, and the so-called 'Ioffe-Pritchard' configuration [38], characterized by a quadratically varying field. The former configuration is the one actually considered by Majorana in his 1932 work. Here, Majorana derives an expression having direct experimental consequences, and clear physical meaning. As Majorana remarks in the Introduction, in the problem of a magnetic dipole adiabatically following the variation of a magnetic field one can immediately recognize two characteristic frequencies: the Larmor frequency, associated with the dipole precession, and the frequency associated with the rotation of the magnetic field, as seen by the moving atom. When the two frequencies become comparable with each other, there will be a relatively high probability for the dipole to change its magnetic sublevel, i.e. to undergo a so-called spin flip. Majorana makes then use of the ratio $k$ between the two frequencies above to scale his dynamical equations, thus showing that for a generic atom the spin flip probability equals $e^{-k \pi / 2}$. It is then evident that a spinflipped atom is now antiparallel with respect to the magnetic field. It will then experience a force which drags it away from the magnetic field zero. In order to trap atoms, therefore, Majorana's spin flip is rather associated with loss of trapped atoms. The problem is alleviated with a Ioffe-Pritchard configuration, since then there is no magnetic field zero, and the rotation of the field as seen by the moving atoms is then usually slower than Larmor frequency.

In the following, we describe an experiment carried out by the students of the Scuola Superiore of Catania to exhibit such a phenomenon. Rubidium atoms have been cooled down to $\sim 10 \mu \mathrm{K}$ and trapped in different magnetic field configurations, as generated by microstructured conductors in a so-called atom chip [39]. The average lifetime of the atoms in a trap has been measured in both a quadrupole and in a Ioffe-Pritchard configuration, thereby allowing to test their difference against the estimate according to Majorana's expression.

Let $G$ be the magnetic field gradient. For an atom passing with velocity $v$ at a distance $d$ from a zero in the magnetic field, one then has

$$
k=\frac{g \mu_{\mathrm{B}}}{\hbar} G \frac{d^{2}}{v}
$$

where $g$ is the magnetic moment of the atom under consideration and $\mu_{\mathrm{B}}$ is Bohr's magneton. Setting $k=1$ one can obtain the value $d_{\text {sf }}$ of distance $d$ at which the spin flip probability will be $50 \%$. One can then estimate that nearly half the atoms entering in a volume of the order of $d_{\mathrm{sf}}^{3}$ per unit time will be lost by the trap. Atoms having a thermal velocity $v$ trapped in a quadrupole configuration will occupy a volume roughly equal to $d_{T}^{3}$, with

$$
d_{T}=\frac{m}{g \mu_{\mathrm{B}} G} v^{2},
$$

where $m$ denotes the mass of an atom. The average lifetime $\tau$ due to spin flip losses can then be approximated by

$$
\tau=2 \frac{d_{\mathrm{sf}}}{v}\left(\frac{d_{T}}{d_{\mathrm{sf}}}\right)^{3} .
$$



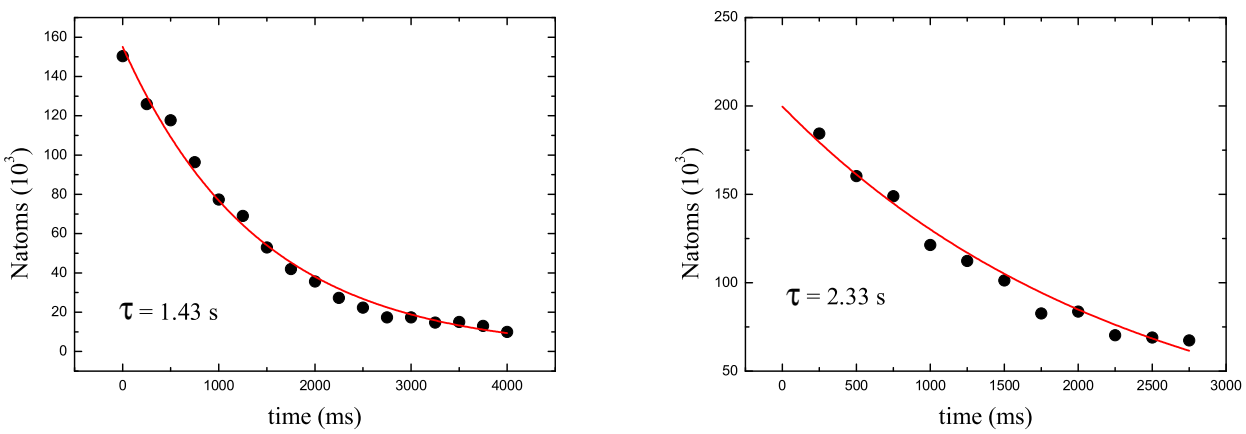

Figure 4: (Color online.) Left: Rubidium atoms at $38 \pm 5 \mu \mathrm{K}$ in a quadrupole trap at a distance $\sim 350 \mu \mathrm{m}$ below a wire with a current of $2 \mathrm{~A}$. The magnetic field gradient equals $\sim 3.3 \mathrm{~T} / \mathrm{m}$. Right: Rubidium atoms at $42 \pm 5 \mu \mathrm{K}$ in a Ioffe-Pritchard trap at a distance $\sim 350 \mu \mathrm{m}$ below a wire with a current of $2 \mathrm{~A}$. Red solid lines are a best-fit to the experimental points with an exponential function.

For atoms at a temperature $T=40 \mu \mathrm{K}$ in the presence of a gradient $G \simeq 3 \mathrm{~T} / \mathrm{m}$ one obtains $\tau=0.8 \mathrm{~s}$, which is a rather crude estimate of the actual value. At the same level of approximation, we can roughly assume that losses due to spin flip are absent in a Ioffe-Pritchard configuration. In such a case, then, the lifetime will be determined solely by losses due to collisions between trapped atoms and atoms at ambient temperature which are present in the surrounding environment. ${ }^{2}$ Fig. 4 plots lifetime measurements in the two configurations for atoms with $T=38 \pm 5 \mu \mathrm{K}$ in a magnetic field gradient $G \simeq 3.3 \mathrm{~T} / \mathrm{m}$. The lifetimes of the atoms in the two different configurations differ by $0.9 \mathrm{~s}$, in good agreement with the theoretical estimate of $0.8 \mathrm{~s}$. Despite the approximations employed above, the estimate following Majorana's theory already contains all the relevant physical aspects of the problem.

\section{Helium molecular ion}

In his successive work [8], Majorana deals with the formation of the molecular ion $\mathrm{He}_{2}^{+}$. There again, Majorana demonstrates his exceptional ability to focus on the main physical aspects of the problem, while showing the limitations of his own theoretical approximations. He immediately observes that the problem is more similar to the formation of the molecular ion $\mathrm{H}_{2}^{+}$, than to the reaction $\mathrm{He}+\mathrm{H}$. The most relevant forces, especially close to the equilibrium distance, are therefore the resonance forces, rather than the polarization ones. By exchanging the two nuclei, the system remains unchanged. Majorana makes then use of the method of Heitler and London [40], and emphasizes the importance of inversion symmetry with respect to the middle point between the nuclei, set at a distance $R$ apart.

Heitler and London [40] introduced a relatively simple expression for the wave-function $\Psi$ of the two electrons in a hydrogen molecule $\mathrm{H}_{2}$ in terms of the wave-functions $\varphi$ and $\psi$ of one electron in the atomic orbital corresponding to atom $a$ and $b$, respectively:

$$
\Psi(1,2)=\varphi(1) \psi(2) \pm \psi(1) \varphi(2)
$$

\footnotetext{
${ }^{2}$ Atom cooling experiments take place at about $10^{-7} \mathrm{~Pa}$.
} 
where 1 and 2 denote the coordinates of the two electrons, respectively. The wave-function $\Psi_{S}$, corresponding to the choice of the plus sign in Eq. (4.1), is symmetric with respect to the exchange of the coordinates of both electrons and nuclei, while $\Psi_{A}$ (minus sign in Eq. (4.1)) is antisymmetric. The full wave-function is globally anti-symmetric, but here we are neglecting its spin part, since the Hamiltonian is spin independent.

As in Heitler-London, in order to study the case of $\mathrm{He}_{2}^{+}$, Majorana starts from the asymptotic solution, namely for large values of $R$. In the case of $\mathrm{H}_{2}$, for large values of $R$, it is very unlikely that both electrons reside on the same nucleus. Similarly, in the case of $\mathrm{He}_{2}^{+}$, Majorana neglects the possibility that all three electrons be located on the same nucleus. Ettore then proceeds by writing the unperturbed eigenfunctions for the three electrons (labeled 1, 2, 3 below) in $\mathrm{He}_{2}^{+}$as

$$
\begin{array}{ll}
A_{1}=\varphi_{1} \Psi_{23}, & B_{1}=\psi_{1} \Phi_{23} \\
A_{2}=\varphi_{2} \Psi_{31}, & B_{2}=\psi_{2} \Phi_{31} \\
A_{3}=\varphi_{3} \Psi_{12}, & B_{3}=\psi_{3} \Phi_{12}
\end{array}
$$

where $\Phi$ and $\varphi$ denote the wave-functions of the neutral and ionized $a$ atom, respectively, while $\Psi$ and $\psi$ denote the analogous wave-functions for atom $b$. Evidently, $A_{2}$ and $A_{3}$ can be obtained from $A_{1}$ by permuting the electrons, and the $B$ 's from the $A$ 's by exchanging the nuclei.

The interaction between the atoms mixes all these wave-functions, but by means of general symmetry considerations, first introduced by Hund [41], as well as of inversion symmetry and of Pauli exclusion principle, Majorana concludes that the only acceptable wave-functions are

$$
\begin{aligned}
& y_{1}=A_{1}-A_{2}+B_{1}-B_{2}, \\
& y_{2}=A_{1}-A_{2}-B_{1}+B_{2},
\end{aligned}
$$

which are antisymmetric in 1 and 2 . In particular, $y_{2}$ corresponds to the $(1 s \sigma)^{2} 2 p \sigma\left({ }^{2} \Sigma\right)$ configuration, viz. the bonding solution for the $\mathrm{He}_{2}^{+}$molecular ion. The latter configuration is characterized by two electrons in the $\sigma$ molecular orbital built from the two $1 s$ atomic orbitals, one electron in the $\sigma$ molecular orbital built from the $2 p$ atomic orbitals, as well as by a value of the total orbital angu$\operatorname{lar}$ momentum $L=0$, and by a value of the total spin $S=+\frac{1}{2}$. The wave-function Eq. (4.3b) clearly shows that the ground state is a resonance between the configurations $\mathrm{He}$ : $-\mathrm{He}$ - and $\mathrm{He} \cdot-\mathrm{He}$, where each dot denotes the presence of one electron on the $a$ or $b$ atom.

In order to perform the calculation of the interaction terms explicitly, making use of analytic expressions, one can take the ground state of the helium atom as the product of two hydrogenoid wave-functions. However, it is well known that the result is greatly improved if, instead of taking the bare charge $Z=2$ of the He nucleus, an effective nuclear charge $Z_{\text {eff }}$ is introduced, to be determined variationally. The fundamental effect here taken into account by Majorana is that of screening: In an atom with many electrons, each electron sees the nuclear charge $Z e$ as slightly attenuated by the presence of the remaining electrons.

The concept of an effective nuclear charge, already introduced for the helium atom, had been extended by Wang [42] to the hydrogen molecule. Probably Majorana was not aware of Wang's work, since he does not refer to it in his 1931 paper. In any case, Majorana is the first one to make 


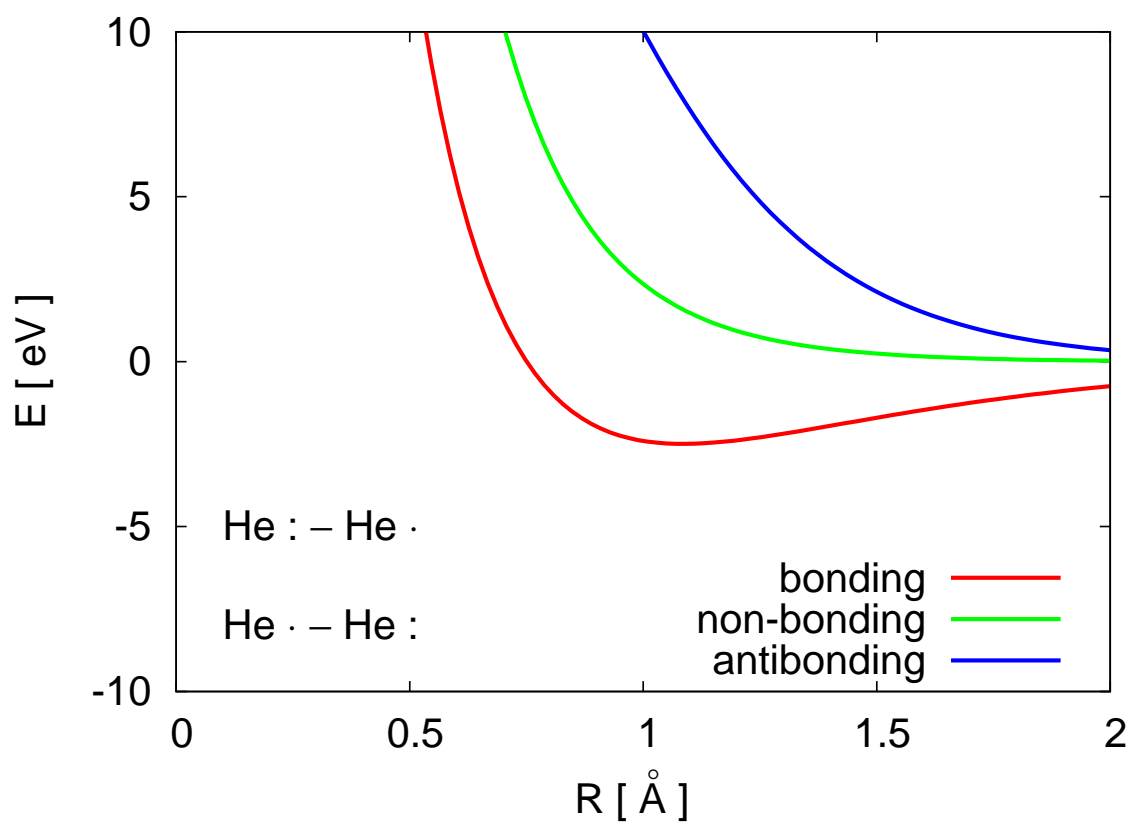

Figure 5: (Color online.) Variational energies of the molecular ion $\mathrm{He}_{2}^{+}$, as a function of the internuclear distance $R$. Red line refers to the symmetric wave-function in Eq. (4.1), blue line to the antisymmetric one, while green line refers to the 'non-bonding' case, where position exchange is neglected. Redrawn after Ref. [43] (see also Ref. [45]).

use of such a method for $\mathrm{He}_{2}^{+}$. In making reference to his own work [43], where $Z_{\text {eff }}$ is used as a variational parameter for $\mathrm{He}_{2}^{+}$, Pauling reports in a footnote ${ }^{3}$

"The same calculation with $Z_{\text {eff }}$ given the fixed value 1.8 was made by E. Majorana [8]."

The variational value obtained by Pauling for $Z_{\text {eff }}$ is 1.833 .

By making use of his results, Majorana evaluates the equilibrium internuclear distance as $d=1.16 \AA$, in good agreement with the experimental value $1.087 \AA$. He can then estimate the vibrational frequency as $n=1610 \mathrm{~cm}^{-1}$, which he compares with the experimental value $1628 \mathrm{~cm}^{-1}$. Majorana concludes his paper by stating [8] that his own result is

"casually in perfect agreement with the experimentally determined value"

(our italics). Any other author would have emphasized such a striking agreement as a success of his own method, whereas Majorana rather underlines the drawbacks of his own approximations.

We would like to remind that he also estimates the minimum energy, i.e. the dissociation energy, finding the value $E_{\min }=-1.41 \mathrm{eV}$, but he had no available experimental data to compare with, at that time. However, he is not satisfied with such a result and collects [8]

"all the errors of the method under the words 'polarization forces',"

\footnotetext{
${ }^{3}$ See footnote on p. 359 of Ref. [44].
} 
which he estimates for very distant nuclei using the polarizability of the neutral $\mathrm{He}$ atom. $\mathrm{He}$ then finds $E_{\min }=-2.4 \mathrm{eV}$. More recent theoretical calculations, using the method of configuration interactions [46] or ab initio variational methods [47], have estimated the value $E_{\min }=-2.47 \mathrm{eV}$. The experimental value has been accurately determined quite recently [48] as $E_{\min }=-2.4457 \pm$ $0.0002 \mathrm{eV}$. We are not claiming that Ettore's result is more accurate than the theoretical results mentioned above. However, he certainly understood the essential physical effects for that system, and made use of appropriate approximations to estimate them. In particular, it is interesting how he emphasizes the quest for the symmetries of the system (see the translation of a paper by Majorana in Ref. [23]). As in the case of $\mathrm{H}_{2}$, also for $\mathrm{He}_{2}^{+}$it is essential to include the position exchange term between $\mathrm{He}$ and $\mathrm{He}^{+}$, in order to have chemical bonding, as it can be seen in Fig. 5, redrawn after Ref. [45]. If one had neglected the resonance $\mathrm{He}: \mathrm{He}^{+} \rightleftharpoons \mathrm{He}^{+}: \mathrm{He}$ (see dashed line in Fig. 5), chemical bonding would have been impossible.

\section{The discovery of the neutron}

Rutherford's pioneering work [49] paved the way not only to Bohr's atomic model, but also to nuclear physics.

In 1930 Bothe and Becker [50], like Rutherford, employed $\alpha$ particles against a berillium target in a scattering experiment. They observed the emission of a very penetrating radiation, which they interpreted as $\gamma$ rays. In successive experiments, Irène Curie and Frederic Joliot [51, 52], her husband, developed further these experiments, but they arrived at similar conclusions. According to Emilio Segrè's account [53], Majorana thus commented the Joliots' results:

"They haven't realized they have discovered the neutral proton."

At this point we should remind that at that time it was believed that the nucleus was composed by protons and electrons. It was Chadwick [54] who soon after demonstrated that the radiation emitted in the Joliots' experiments was made up by neutral particles, whose mass is very close to the proton's mass. It was probably Fermi [53] who first distinguished between the neutrinos conjectured by Pauli, and the neutrons discovered by Chadwick.

Meanwhile, Majorana developed a theory of the nucleus containing protons and neutrons and then, according to Segrè [53],

"he analyzed, as far as it was possible, the nuclear forces on the basis of the available experimental results, and he estimated the binding energies of the lightest nuclei. When he presented his work to Fermi and ourselves, we immediately recognized its importance. Fermi encouraged Majorana to publish his own results, but Majorana refused to do so, saying they were yet too incomplete."

More than that, when Fermi asked Majorana whether he could make reference to his results during a forthcoming conference in Paris, Ettore mockingly replied he would agree, provided the reference was attributed to an old professor of electrochemistry, who was also going to attend the same conference. Obviously, Fermi could not accept Majorana's condition, and no reference was then made to his results during the conference. 

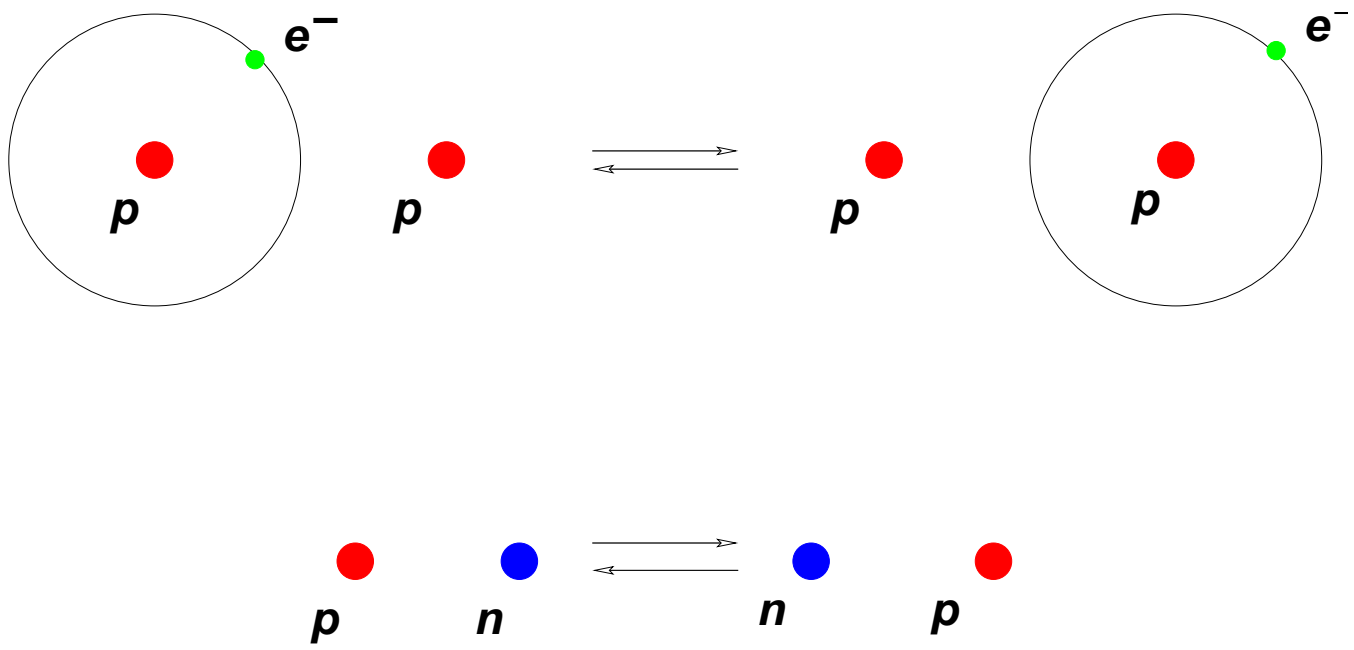

Figure 6: (Color online.) Exchange interactions. Resonant forms in the hydrogen molecular ion, $\mathrm{H}: \mathrm{H}^{+} \rightleftharpoons$ $\mathrm{H}^{+}: \mathrm{H}$ (upper row), and in the proton-nucleon pair inside a nucleus, $p: n \rightleftharpoons n: p$ (lower row).

Meanwhile, people started feeling the lack of a theory of nuclear forces, conveniently taking into account for the presence of both protons and neutrons in the nucleus. But where to begin with?

\section{Heisenberg-Majorana forces}

To this aim, in three fundamental contributions [55, 56, 57], Heisenberg assumed hydrogen molecular ion $\mathrm{H}_{2}^{+}$as a model. He recognizes that the most important nuclear forces are not the polarization forces among the neutrons, or Coulombic repulsion among protons, but the exchange forces between protons and neutrons.

Heisenberg emphasizes that neutrons obey to Fermi statistics. Moreover, since a neutron possesses spin $\frac{1}{2} \hbar$, it cannot be simply thought of as composed of a proton plus an electron, unless the latter has zero spin, when inside a neutron. ${ }^{4}$ A neutron is an elementary particle per se. The interactions postulated by Heisenberg are characterized by the exchange of both position coordinates and spins of the two nucleons.

Similarly, Majorana assumed that the fundamental nuclear forces are of exchange nature between protons and neutrons. However, he fully exploits the analogy with $\mathrm{H}_{2}^{+}$(see Fig. 6), regardless of $\operatorname{spin}^{5}$

Let $\mathbf{r}_{1}, \sigma_{1}$ and $\mathbf{r}_{2}, \sigma_{2}$ denote the position and spin coordinates of the first and the second nucleon, respectively, and let $\psi\left(\mathbf{r}_{1}, \sigma_{1} ; \mathbf{r}_{2}, \sigma_{2}\right)$ be the wave-function for a given nucleon pair [59]. Then Heisenberg exchange $P^{H}$ implies

$$
P^{H} \psi\left(\mathbf{r}_{1}, \sigma_{1} ; \mathbf{r}_{2}, \sigma_{2}\right)=\psi\left(\mathbf{r}_{2}, \sigma_{2} ; \mathbf{r}_{1}, \sigma_{1}\right),
$$

\footnotetext{
${ }^{4}$ Besides considerations concerning the spin, such a model would require an enormous amount of energy to localize the electron within the neutron [56].

${ }^{5}$ Current literature usually employs the formalism of isotopic spin to describe the exchange character of the nuclear forces. However, as noted by Blatt and Weisskopf [59], this is equivalent to a description which makes use of the forces of Bartlett, Heisenberg, Majorana, and Wigner.
} 


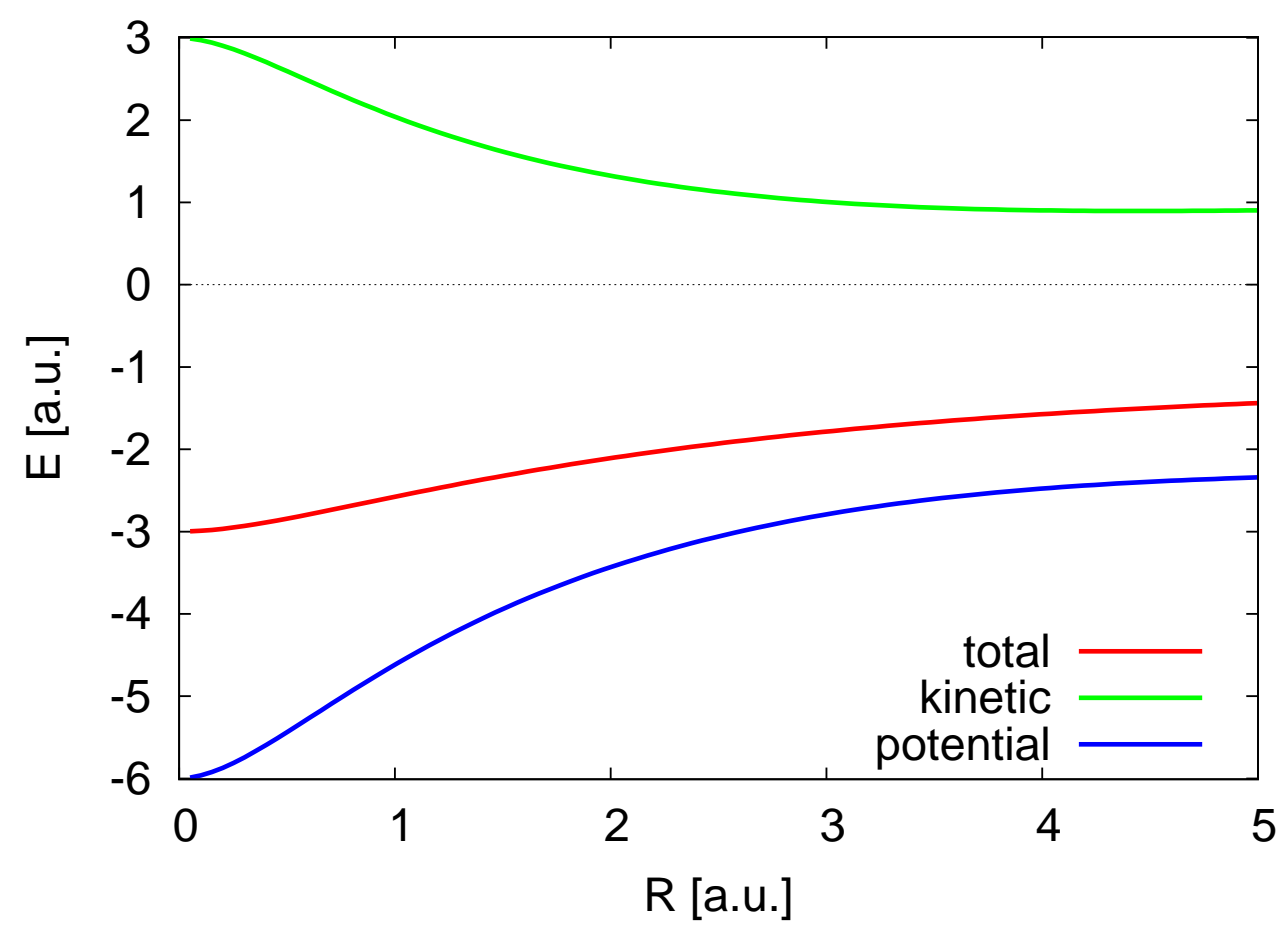

Figure 7: (Color online.) Kinetic, potential, and total energies for the ground state of $\mathrm{H}_{2}^{+}$, excluding nuclear repulsion, within the linear combination of atomic orbitals (LCAO) approximation. Cf. Fig. 2.4 in Ref. [58], where the same quantities have been obtained within a variational method.

whereas Majorana exchange $P^{M}$ implies

$$
P^{M} \psi\left(\mathbf{r}_{1}, \sigma_{1} ; \mathbf{r}_{2}, \sigma_{2}\right)=\psi\left(\mathbf{r}_{2}, \sigma_{1} ; \mathbf{r}_{1}, \sigma_{2}\right)
$$

In Majorana's own notation (apart from a minus sign here included in the definition of $J(r)$ ), the exchange interaction then reads [2]

$$
\left(Q^{\prime}, q^{\prime}|J| Q^{\prime \prime}, q^{\prime \prime}\right)=J(r) \delta\left(q^{\prime}-Q^{\prime \prime}\right) \delta\left(q^{\prime \prime}-Q^{\prime}\right)
$$

where $Q$ and $q$ are the position coordinates of the neutron and the proton, respectively, and $r=$ $\left|q^{\prime}-Q^{\prime}\right|$ is their relative distance. Majorana then plots a qualitative sketch of $J(r)$ (cf. Fig. 2 in Ref. [2]), which closely resembles the behaviour of the potential energy in $\mathrm{H}_{2}^{+}$, when the internuclear repulsion is neglected (Fig. 7).

In the same paper [2], in addition to his knowledge of molecular physics, Majorana fully exploits also his acquaintance with the atomic statistical model. Indeed, he defines the nuclear density as

$$
\rho=\frac{8 \pi}{3 h^{3}}\left(P_{n}^{3}+P_{p}^{3}\right)
$$

in complete analogy with Eq. (2.1), where $P_{n}$ and $P_{p}$ are the Fermi momenta of neutrons and protons, respectively. From this model, he derives an asymptotic expression $(\rho \rightarrow \infty)$ for the exchange energy per particle,

$$
\left.a(\rho)\right|_{\rho \rightarrow \infty}=-\frac{n_{2}}{n_{1}+n_{2}} J(0),
$$


where $n_{1}$ and $n_{2}$ are the numbers of neutrons and protons, respectively. As in Thomas-Fermi model, the kinetic energy per particle, $t$ say, is given by

$$
t \propto \rho^{2 / 3} .
$$

From the competition between kinetic and potential energy, the total energy attains a minimum as a function of $r$ (cf. Fig. 1 in Ref. [2]).

Majorana's model explains two fundamental properties of nuclear physics [59]: (a) the density of nucleons is about the same for all nuclei (density saturation); (b) the binding energy per nucleon is about the same for all nuclei (binding energy saturation).

\section{Concluding remarks}

Majorana's legacy to contemporary physics can be ultimately summarized in the quest for symmetry in Nature and its laws.

It was the quest for symmetry (between space and time) which led Einstein to the formulation of special relativity, and it was the unification between wave and particle behaviours which led most distinguished physicists at the beginning of the 20th century (including Einstein himself and de Broglie, among the others) to the development of quantum mechanics. The same quest for symmetry, but now in matter at various energy scales (molecular, atomic, nuclear, and subnuclear), was the main guiding line of Majorana's research, as is exemplified by his treatment of ThomasFermi model.

Due to his hypercritical spirit, Majorana was probably unsatisfied with the asymptotic behaviour of the self-consistent potential $\phi$ in Thomas-Fermi model, as it seems to emerge from a short note [7]. In such a short contribution, Majorana demonstrates not only his unconventional mathematical skills, but also his ability to catch the physical essence of a problem. His attention to asymptotic properties and to symmetry is also clearly expressed in his paper on $\mathrm{He}_{2}^{+}$molecular ion [8].

We have emphasized that exchange symmetry is essential for the formation of the chemical bond, which inspired Majorana his model of nuclear forces.

The tremendous importance given by Majorana to the quest of symmetry clearly emerges in his celebrated work on the neutrino [3]. There, by imposing severe symmetry constraints since the outstart, he finds different properties for the neutrino, than those predicted by Dirac. The relevance of such a problem within the physics of elementary particles provides continuous motivation to an intense research activity, both from the theoretical and from the experimental point of view $[5,60,61,62]$.

Neutrinos are also relevant to astrophysics and cosmology [63], where the attempt at a consistent unification of quantum mechanics and general relativity seems to indicate the necessity to go beyond the Standard Model. Preliminary attempts in this direction are provided by the string theory [64] as well as by loop quantum gravity [65, 66].

On the other hand, Majorana's theoretical works on atomic and molecular physics continue to suggest experiments, which were not feasible in Majorana's times. The development in the field of Bose-Einstein condensation of atomic gases confined in magnetic traps has recently enabled to 
carry out experiments implicitly suggested by Majorana's paper [9] on the behaviour of an atom in a varying magnetic field.

\section{Acknowledgements}

We are grateful to M. Baldo, L. Paternò, G. Piccitto for useful comments and discussions, and to E. Rimini and M. Inguscio for their continuous support. We would also like to thank N. H. March for several stimulating discussions and for collaboration over the general area embraced by this article. Thanks are also due to I. Herrera, A. Mio, M. Siciliani de Cumis for the experimental figures.

\section{References}

[1] E. Majorana, Nuovo Cimento 9, 335 (1932).

[2] E. Majorana, Z. Physik 82, 137 (1933).

[3] E. Majorana, Nuovo Cimento 14, 171 (1937).

[4] E. Amaldi, in Strong and Weak Interactions. Present problems, edited by A. Zichichi (Academic Press, New York, 1966), p. 10.

[5] P. Sapienza for the NEMO collaboration, Nucl. Phys. B: Proc. Suppl. 145, 331 (2005).

[6] A. Bettini, Fisica subnucleare (Università degli Studi di Padova, Padova, 2004), available at http://www.pd.infn.it/〜bettini.

[7] E. Majorana, Nuovo Cimento 6, xiv (1929).

[8] E. Majorana, Nuovo Cimento 8, 22 (1931).

[9] E. Majorana, Nuovo Cimento 9, 43 (1932).

[10] E. Amaldi, Giornale di Fisica 9, 300 (1968).

[11] Ettore Majorana: Lezioni all'Università di Napoli, edited by B. Preziosi (Bibliopolis, Napoli, 1987).

[12] L. Bonolis, Majorana, il genio scomparso (Le Scienze, Milano, 2002).

[13] E. Recami, Quaderni di Storia della Fisica del Giornale di Fisica 5, 19 (1999), also available as preprint arXiv:physics/9810023.

[14] A. Einstein, Ann. Physik 17, 891 (1905).

[15] A. Einstein, Ann. Physik 17, 132 (1905).

[16] L. de Broglie, The Revolution in Physics (Grossett and Dunlop, New York, 1955).

[17] H. A. Bethe and N. D. Mermin, Phys. Today 57, 53 (2004).

[18] L. H. Thomas, Proceedings of the Cambridge Philosophical Society, Mathematical and Physical Sciences 23, 542 (1926).

[19] E. Fermi, Rendiconti dell'Accademia Nazionale dei Lincei 6, 602 (1927).

[20] N. H. March, Self-Consistent Fields in Atoms (Pergamon Press, Oxford, 1975).

[21] A. Sommerfeld, Rend. R. Accademia dei Lincei 15, 293 (1932).

[22] S. Esposito, Am. J. Phys. 70, 852 (2002). 
[23] S. Esposito, E. Majorana Jr., A. van der Merwe, and E. Recami, Ettore Majorana: Notes on Theoretical Physics (Kluwer, New York, 2003).

[24] E. Di Grezia and S. Esposito, Foundations of Physics 34, 1431 (2004).

[25] F. Guerra and N. Robotti, (2005), preprint arXiv:physics/0511222.

[26] R. Pucci and G. G. N. Angilella, Found. Phys. 36, ... (2006), preprint arXiv:physics / 0605226.

[27] E. Fermi, Mem. Accad. Italia (Fis.) I, 139 (1930).

[28] S. Esposito, (2005), preprintarXiv:physics/0512259

[29] S. Flügge, Practical Quantum Mechanics (Springer, New York, 1974).

[30] E. Fermi and E. Amaldi, Mem. Accad. Italia (Fis.) 6, 119 (1934).

[31] R. Pucci and N. H. March, J. Chem. Phys. 76, 4089 (1982).

[32] W. Gerlach and O. Stern, Z. Physik 9, 349 (1922).

[33] T. E. Phipps and O. Stern, Z. Physik 73, 185 (1932).

[34] P. Güttinger, Z. Physik 73, 169 (1932).

[35] E. A. Cornell and C. E. Wieman, Rev. Mod. Phys. 74, 875 (2002).

[36] W. Ketterle, Rev. Mod. Phys. 74, 1131 (2002).

[37] F. Bloch and I. I. Rabi, Rev. Mod. Phys. 17, 237 (1945).

[38] D. E. Pritchard, Phys. Rev. Lett. 51, 1336 (1983).

[39] R. Folman, P. Krüger, D. Cassettari, B. Hessmo, Th. Maier, and J. Schmiedmayer, Phys. Rev. Lett. 84, 4749 (2000).

[40] W. Heitler and F. London, Z. Physik 44, 455 (1927).

[41] F. Hund, Z. Physik 43, 788 (1927).

[42] S. C. Wang, Phys. Rev. 31, 579 (1928).

[43] L. Pauling, J. Chem. Phys. 1, 56 (1933).

[44] L. Pauling and E. Bright Wilson, Introduction to Quantum Mechanics with Applications to Chemistry (McGraw-Hill, New York, 1935).

[45] L. Pauling, L. O. Brockway, and J. Y. Beach, J. Am. Chem. Soc. 57, 2705 (1935).

[46] J. Ackermann and H. Hogreve, Chem. Phys. 157, 75 (1991).

[47] P. N. Reagan, J. C. Browne, and F. A. Matsen, Phys. Rev. 132, 304 (1963).

[48] L. Coman, M. Guna, L. Simons, and K. A. Hardy, Phys. Rev. Lett. 83, 2715 (1999).

[49] E. Rutherford, Collected papers (Interscience: J. Wiley \& Sons, New York, 1963).

[50] W. Bothe and H. Becker, Z. Physik 66, 289 (1930).

[51] I. Joliot-Curie and F. Joliot, Compt. Rend. 194, 273 (1932).

[52] I. Joliot-Curie and F. Joliot, Compt. Rend. 194, 876 (1932).

[53] E. Segrè, Enrico Fermi, Physicist (The University of Chicago Press, Chicago, 1970).

[54] J. Chadwick, Nature 129, 312 (1932). 
[55] W. Heisenberg, Z. Physik 77, 1 (1932).

[56] W. Heisenberg, Z. Physik 78, 156 (1933).

[57] W. Heisenberg, Z. Physik 80, 587 (1933).

[58] J. C. Slater, Quantum theory of molecules and solids (McGraw-Hill, New York, 1963), Vol. 1.

[59] J. M. Blatt and V. F. Weisskopf, Theoretical Nuclear Physics (J. Wiley \& Sons, New York, 1952).

[60] A. Guglielmi and P. R. Sala, Le Scienze 454, 62 (2006).

[61] H. V. Klapdor-Kleingrothaus, I. V. Krivosheina, A. Dietz, and O. Chkvorets, Phys. Lett. B 586, 198 (2004).

[62] Cf. the website of the INFN Labs at Gran Sasso, http://wWw. Ings . infn.it

[63] S. W. Allen, R. W. Schmidt, and S. L. Bridle, Mon. Not. Roy. Astron. Soc. 346, 593 (2003).

[64] B. Greene, L'Universo elegante (Einaudi, Torino, 2003).

[65] L. Crane, A. Perez, and C. Rovelli, Phys. Rev. Lett. 87, 181301 (2001).

[66] C. Rovelli, Quantum Gravity (Cambridge University Press, Cambridge, 2004). 